\title{
Melatonin inhibits angiogenesis in SH-SY5Y human neuroblastoma cells by downregulation of VEGF
}

\author{
ALICIA GONZÁLEZ, ALICIA GONZÁLEZ-GONZÁLEZ, CAROLINA ALONSO-GONZÁLEZ, \\ JAVIER MENÉNDEZ-MENÉNDEZ, CARLOS MARTÍNEZ-CAMPA and SAMUEL COS
}

\author{
Department of Physiology and Pharmacology, School of Medicine, University of Cantabria \\ and Valdecilla Research Institute (IDIVAL), ES-39011 Santander, Spain
}

Received August 11, 2016; Accepted October 10, 2016

DOI: $10.3892 /$ or.2017.5446

\begin{abstract}
Vascular endothelial growth factor (VEGF) produced from tumor cells plays a crucial role in the pathogenesis and neovascularization of neuroblastoma. Inhibition of VEGF secretion by tumor cells, as well as VEGF-regulated signaling in endothelial cells, are important to reduce the angiogenesis and growth of neuroblastoma. Since melatonin has anti-angiogenic effects in tumor cell lines, the aim of the present study was to study melatonin modulation of the pro-angiogenic effects of VEGF in neuroblastoma cells (SH-SY5Y). We used co-cultures of SH-SY5Y and endothelial cells. VEGF expression and protein levels were analyzed by quantitative RT-PCR and ELISA, respectively. Endothelial cell migration was assessed by wound-healing assay and endothelial angiogenesis by a tube formation assay. Melatonin inhibited the pro-angiogenic effects of SH-SY5Y cells. The conditioned medium collected from the neuroblastoma cells was angiogenically active and stimulated proliferation, migration and tube formation in endothelial cells. This effect was significantly counteracted by the addition of either anti-VEGF or melatonin. Melatonin inhibited VEGF expression and secretion in SH-SY5Y cells, decreasing the levels of VEGF available for endothelial cells. Melatonin has anti-angiogenic effects at different steps of the angiogenic process in SH-SY5Y neuroblastoma cells, through the downregulation of VEGF.
\end{abstract}

\section{Introduction}

Angiogenesis is the process of the development of the intrinsic vascular network, and it is an essential event in the progression and metastatic spread of solid tumors such as

Correspondence to: Dr Samuel Cos, Department of Physiology and Pharmacology, School of Medicine, University of Cantabria and Valdecilla Research Institute (IDIVAL), ES-39011 Santander, Spain E-mail: coss@unican.es

Key words: melatonin, pineal gland, neuroblastoma cells, angiogenesis, SH-SY5Y cells, endothelial cells, VEGF neuroblastoma, where new capillaries spread from preexisting vessels and the transition from avascular to vascular phase occurs via neovascularization (1). Angiogenesis is mediated by multiple regulatory factors such as growth factors, adhesion molecules and matrix degrading enzymes. The balance between angiogenic activators and inhibitors maintains endothelial cells in an angiogenic or quiescent stage (2). Malignant neuroblastoma is a highly vascularized solid tumor that requires access to blood vessels for growth, invasion and metastasis, being highly dependent on angiogenesis (1). Therefore, anti-angiogenic strategies can be effective in inhibiting tumor cell dissemination and metastasis in highly vascular neuroblastoma $(3,4)$. Vascular endothelial growth factor (VEGF) is the most active endogenous pro-angiogenic factor, and it can induce angiogenesis by directly acting on the endothelium in vivo as well as by increasing the microvascular permeability (5). VEGF is widely known to play an important role in tumor biology and more specifically, in the process of tumor angiogenesis, as VEGF expression plays a crucial role in the pathogenesis and neovascularization of neuroblastoma (1). VEGF signaling plays a regulatory role in neuroblastoma angiogenesis via a paracrine mechanism through two specific tyrosine kinase VEGF receptors, VEGFR-1 and VEGFR-2, located at the surface of endothelial cells. Tumor-induced VEGF secretion may function in both paracrine and autocrine manners to allow tumor expansion and growth (6). Treatment of neuroblastoma in both in vitro and in vivo models with an anti-VEGF agent results in antitumor activity with a decrease in both tumor vascularity and in the number of intratumoral vessels and a decrease in VEGF expression suggesting a diversified role of VEGF in the progression of advanced stage neuroblastoma $(7,8)$.

Melatonin, the main secretory product of the pineal gland, is widely known to decrease the growth and development of estrogen-responsive breast cancers (9-12). Melatonin exerts its oncostatic properties in hormone-dependent breast cancer by interfering at different levels with the estrogen signaling pathways (13-15). Melatonin downregulates circulating levels of gonadal estrogens, interferes with the activation of the estrogen receptor and regulates the activity and expression of several enzymes involved in the biosynthesis of estrogens in tumoral and peritumoral tissues (16-19). In vitro studies demonstrated that melatonin plays a role in 
the paracrine interactions between malignant epithelial and proximal endothelial cells, through the downregulation of VEGF expression in human breast cancer cells, decreasing the levels of VEGF around endothelial cells and decreasing angiogenesis. VEGF secreted by breast cancer cells interacts with VEGF receptors in endothelial cells and stimulates downstream signaling molecules to promote proliferation, growth, survival and migration of endothelial cells (20-22). Therefore, inhibition of VEGF secretion by tumor cells, as well as VEGF-regulated signaling in endothelial cells, could be important in decreasing tumor angiogenesis and growth.

Since melatonin has anti-angiogenic effects in other tumor cell lines (23), in the present study, we investigated the anti-angiogenic activity of melatonin at different steps of the angiogenic process in SH-SY5Y neuroblastoma cells. We studied whether melatonin decreases the pro-angiogenic effects of VEGF, as well as the conditioned media from SH-SY5Y neuroblastoma cells. To accomplish this, we used cultures of neuroblastoma cells (SH-SY5Y) and co-cultures of SH-SY5Y cells with human umbilical vein endothelial cells (HUVECs).

\section{Materials and methods}

Cells and culture conditions. HUVECs were purchased from the American Type Culture Collection (ATCC; Rockville, MD, USA). They were maintained as monolayer cultures in $75 \mathrm{~cm}^{2}$ plastic culture flasks containing Vascular Cell Basal Medium (VCBM) supplemented with endothelial cell growth kit-BBE (both from ATCC) which consisted of $2 \%$ fetal bovine serum (FBS) (PAA Laboratories, Pasching, Austria), 0.2\% bovine brain extract, $5 \mathrm{ng} / \mathrm{ml} \mathrm{rhEGF}, 10 \mathrm{mM}$ L-glutamine, $0.75 \mathrm{U} / \mathrm{ml}$ heparin sulfate, $1 \mu \mathrm{g} / \mathrm{ml}$ hydrocortisone hemisuccinate, $50 \mu \mathrm{g} / \mathrm{ml}$ ascorbic acid, penicillin $(20 \mathrm{U} / \mathrm{ml})$ and streptomycin $(20 \mu \mathrm{g} / \mathrm{ml})$ (Sigma-Aldrich, Madrid, Spain), at $37^{\circ} \mathrm{C}$ in a humid atmosphere containing $5 \% \mathrm{CO}_{2}$. To avoid genetic mutation and low viability, no more than 6 passages of HUVECs were used for the experiments.

SH-SY5Y neuroblastoma cells were a kind gift from Dr María Elsa Valdizán, from the Pharmacology Division of the Department of Physiology and Pharmacology of the University of Cantabria (Spain). They were maintained as monolayer cultures in $75 \mathrm{~cm}^{2}$ plastic culture flasks in Dulbecco's modified Eagle's medium (DMEM)/Ham's Nutrient Mixture F-12 (Sigma-Aldrich), supplemented with 10\% FBS, penicillin $(20 \mathrm{U} / \mathrm{ml})$ and streptomycin $(20 \mu \mathrm{g} / \mathrm{ml})$ at $37^{\circ} \mathrm{C}$ in a humid atmosphere containing $5 \% \mathrm{CO}_{2}$.

Conditioned media collection for experimentation. SH-SY5Y cells were seeded into 24 -multiwell plates $\left(25 \times 10^{4}\right.$ cells/well $)$ in DMEM/Ham's Nutrient Mixture F-12 supplemented with $10 \% \mathrm{FBS}$ and incubated at $37^{\circ} \mathrm{C}$. When a homogenous monolayer of pre-confluent SH-SY5Y cells was reached (48 h), the cells were washed twice with PBS and placed in serum-free VCBM. After $24 \mathrm{~h}$ of incubation, the conditioned medium was collected, filtered through a $0.2-\mu \mathrm{m}$ pore size membrane to remove particles and stored at $-80^{\circ} \mathrm{C}$.

Cell proliferation assay. SH-SY5Y were seeded into 96-multiwell plates at a density of $25 \times 10^{3}$ cells/well in
DMEM/Ham's Nutrient Mixture F-12 supplemented with $2 \% \mathrm{FBS}$ and incubated at $37^{\circ} \mathrm{C}$. After $24 \mathrm{~h}$ of incubation, the media were aspirated and replaced by fresh media supplemented with 5\% charcoal-stripped FBS (sFBS) containing either $1 \mathrm{mM}$ or $1 \mathrm{nM}$ melatonin (Sigma-Aldrich) or vehicle (ethanol at a final concentration of $<0.0001 \%$ ). Cells were cultured for 6 days and proliferation was assessed using the [3-(4,5-dimethylthiazol-2-yl)-2,5-diphenyltetrazolium bromide] (MTT) method (24), with a reading absorbance at $570 \mathrm{~nm}$ on a microplate reader (Labsystems Multiskan RC 351; Vienna, VA, USA). MTT was obtained from Molecular Probes, Inc. (Eugene, OR, USA).

Paracrine effects of SH-SY5Y conditioned medium on endothelial cell proliferation. HUVECs were seeded at $8 \times 10^{3}$ cells/well in a 96-well multiplate containing VCBM supplemented with $2 \%$ FBS overnight. Cells were washed twice with PBS and the media were replaced with serum-free VCBM containing SH-SY5Y conditioned media alone or supplemented with $1 \mathrm{mM}$ melatonin or $150 \mathrm{ng} / \mathrm{ml}$ anti-VEGF (R\&D Systems Europe Ltd., Abingdon, UK). The cells were then cultured for $24 \mathrm{~h}$ and cellular proliferation was assessed using the MTT method.

Co-culture of HUVECs and SH-SY5Y cells. HUVECs were co-cultured along with SH-SY5Y cells using Falcon 24-multiwell plates and Falcon cell culture inserts. HUVECs were plated $\left(24 \times 10^{3}\right.$ cells/well) on the bottom wells containing VCBM supplemented with 2\% FBS and incubated overnight. Subsequently, SH-SY5Y cells were seeded $\left(35 \times 10^{3}\right.$ cells) on the permeable membrane $(0.45-\mu \mathrm{m})$ of the tissue-culture inserts containing VCBM supplemented with $2 \%$ FBS for $24 \mathrm{~h}$. The media were then changed to serum-free VCBM for $72 \mathrm{~h}$ containing melatonin $(1 \mathrm{mM})$ or vehicle (ethanol). At the end of the experiment, the media were collected, centrifuged to remove particles and subjected to the assessment of the protein levels of VEGF. Cells in the bottom plate were evaluated for proliferative indices by MTT method.

Assessment of VEGF mRNA expression. Analysis of the VEGF expression in SH-SY5Y cells was carried out by RT-PCR after incubation of cells with either $1 \mathrm{mM}, 1 \mu \mathrm{M}$ and $1 \mathrm{nM}$ of melatonin and/or $100 \mu \mathrm{M} \mathrm{CoCl}_{2}$ (Sigma-Aldrich) and/or vehicle (ethanol) for $4 \mathrm{~h}$. Total RNA was isolated from SH-SY5Y cells and purified with the Nucleospin RNA II kit (Machenery-Nagel, Düren, Germany) following the manufacturer's instructions. The absorbance ratio $\mathrm{A}_{260 \mathrm{~nm}} / \mathrm{A}_{280 \mathrm{~nm}}$ was $>1.8$. For cDNA synthesis $0.5 \mu \mathrm{g}$ of total RNA was denatured at $65^{\circ} \mathrm{C}$ for $10 \mathrm{~min}$ and reverse-transcribed for $50 \mathrm{~min}$ at $45^{\circ} \mathrm{C}$ with a cDNA Synthesis kit (BioLine, London, UK) in a final volume of $20 \mu \mathrm{l}$ in the presence of $500 \mathrm{ng}$ of oligo(dT) (12-18 primers). Quantitative real-time (qRT-PCR) was performed using the following set of human VEGF 165 specific primers: [5'-ACCA AGGCCAGCACATAGG-3' (forward) and 5'-ACGCTCCAGG ACTTATACCG-3' (reverse)] (Sigma Genosys Ltd., Cambridge, UK). As a quantification control, s14 mRNA was also subjected to qRT-PCR using a set of specific primers: [5'-TCCTGCGAG TGCTGTCAGAG-3' (forward) and 5'-TCACCGCCCTACAC ATCAAA-3' (reverse)] (Sigma Genosys Ltd.). qRT-PCRs were performed on an MX3005P system (Stratagene, La Jolla, CA, 
USA) using Brilliant ${ }^{\circledR}$ SYBR ${ }^{\circledR}$-Green PCR Master Mix (Applied Biosystems, Madrid, Spain) following the manufacturer's instructions. Amplifications were performed for 40 cycles using the following temperature profile: $60^{\circ} \mathrm{C}, 45 \mathrm{sec}$ (annealing); $72^{\circ} \mathrm{C}, 30 \mathrm{sec}$ (extension) and $95^{\circ} \mathrm{C}, 30 \mathrm{sec}$ (denaturation).

Assessment of the protein levels of VEGF. For the determination of the concentration of VEGF in the SH-SY5Y cell cultures and the HUVEC/SH-SY5Y cell co-culture media, a Human VEGF Immunoassay kit (R\&D Systems Europe Ltd.) was used. The samples (in triplicate) were processed according to the manufacturer's instructions. Briefly, SH-SY5Y cells were seeded into 96 -multiwell plates at a density of $8 \times 10^{3}$ cells/well in DMEM/Ham's Nutrient Mixture F-12 supplemented with $10 \% \mathrm{FBS}$ and incubated at $37^{\circ} \mathrm{C}$ for $24 \mathrm{~h}$. Next, the media were aspirated and replaced by fresh media supplemented with $0.5 \%$ sFBS and containing either $1 \mathrm{mM}$ or $1 \mathrm{nM}$ of melatonin or vehicle (ethanol). After $24 \mathrm{~h}$ of incubation, the media were collected, centrifuged to remove particles and subjected to ELISA according to the manufacturer's instructions. For the determination of the protein levels of VEGF in the cell co-culture media, samples were collected, centrifuged and immediately processed. At the end of the procedure, the absorbance was determined at a wavelength of $450 \mathrm{~nm}$, with the correction wavelength set at $570 \mathrm{~nm}$.

Endothelial cell migration assay: Wound healing assay. HUVECs were seeded onto 6-multiwell plates (Falcon) containing VCBM supplemented with $2 \%$ FBS and were allowed to reach full confluency. A line of HUVECs was then scraped away in each plate using a pipette tip. Subsequently, the cells were washed twice with PBS to remove detached cells and SH-SY5Y conditioned media alone or supplemented with $1 \mathrm{mM}$ melatonin or $150 \mathrm{ng} / \mathrm{ml}$ anti-VEGF were added to each plate. Thereafter, three randomly selected views along the scraped line in each plate were photographed using an ORCA-R2 camera (Hamamatsu Photonics, Massy Cedex, France) attached to a Nikon Eclipse-Ti microscope set (Werfen Group, Barcelona, Spain) at a magnification of x10. Photomicrographs, with an exposure time of $5 \mathrm{msec}$, were captured every 10 min during the course of the experiment, which was terminated as soon as the wound was completely filled in the vehicle-treated controls (after $8 \mathrm{~h}$ ). Initial and final wound sizes were determined using the NIS-Elements v.3.8 software (Nikon, Tokyo, Japan), and the difference between the two wound sizes was used to determine the migratory distance using the following formula: Initial wound size minus final wound size divided by two. Three independent experiments were carried out.

Endothelial cell differentiation assay: Endothelial cell capillary-like tube formation assay. To study the effects of SH-SY5Y conditioned media on the formation of tubular structures in HUVECs we used the In vitro Angiogenesis Assay Tube Formation kit (Cultrex; Trevigen Inc., Gaithersburg, MD, USA) according to the manufacturer's instructions. First, reduced growth factor basement membrane extract (BME) was pippetted into a 24-well plate and polymerized for $1 \mathrm{~h}$ at $37^{\circ} \mathrm{C}$. HUVECs $\left(1 \times 10^{5} /\right.$ well $)$ were then seeded in VCBM supplemented with $2 \%$ FBS containing SH-SY5Y conditioned media alone or supplemented with $150 \mathrm{ng} / \mathrm{ml}$ anti-VEGF or melatonin $1 \mathrm{mM}$. After $5 \mathrm{~h}$ of incubation, 4 random fields were selected/well and tubular structures were photographed using a Nikon Sight DS-SML1 camera (Sendai Nikon Corporation, Miyagi, Japan) attached to a Nikon Eclipse Ti-S fluorescence microscope at a magnification of $\mathrm{x} 10$. The formation of the tubular network was assessed using ImageJ $1.45 \mathrm{~S}$ software. Each assay was performed three times.

Statistical analysis. The data are expressed as the mean \pm standard errors of the mean (SEM). Statistical differences between groups were analyzed using one way analysis of variance (ANOVA), followed by the Student-Newman-Keuls test. Results were considered as statistically significant at $\mathrm{p}<0.05$.

\section{Results}

Effects of melatonin on SH-SY5Y neuroblastoma cell proliferation. To investigate the influence of melatonin on the proliferation of SH-SY5Y cells we studied the effects of physiological $(1 \mathrm{nM})$ and pharmacological $(1 \mathrm{mM})$ concentrations of melatonin on the proliferation of neuroblastoma cells. Only pharmacological concentrations of melatonin $(1 \mathrm{mM})$ had a significant inhibitory effect on cell proliferation (Fig. 1A).

Effects of melatonin on secretion and VEGF $m R N A$ expression in SH-SY5Y cells. First, we studied the effect of melatonin on VEGF synthesis by SH-SY5Y cells. For this purpose, we assessed the VEGF concentration in the SH-SY5Y-cell culture media. The levels of VEGF were significantly $(p<0.001)$ decreased by the addition of melatonin at a $1 \mathrm{mM}$ concentration (Fig. 1B).

With the aim of determining whether this inhibitory effect of melatonin on VEGF production was due to a downregulation of VEGF mRNA expression, we incubated SH-SY5Y cells with melatonin $(1 \mathrm{mM}, 1 \mu \mathrm{M}$ and $1 \mathrm{nM})$ or vehicle for $4 \mathrm{~h}$, and total RNA was isolated to perform RT-PCR with specific primers for human VEGF, using s14 as a control housekeeping gene. Melatonin at different concentrations decreased VEGF mRNA expression (Fig. 2A). To mimick hypoxia, a wellknown inducer of VEGF mRNA expression, SH-SY5Y cells were exposed to $100 \mu \mathrm{M} \mathrm{CoCl}_{2}$ and the addition of melatonin at the pharmacological $(1 \mathrm{mM})$ concentration, significantly counteracted the stimulatory effect of $\mathrm{CoCl}_{2}$ on VEGF mRNA gene expression $(\mathrm{p}<0.001)$ (Fig. $2 \mathrm{~B})$.

We developed co-cultures of HUVECs and SH-SY5Y cells to first investigate whether the presence of neuroblastoma cells in the cultures affects the growth of the endothelial cells. Undoubtedly, we observed that the presence of SH-SY5Y cells in the co-cultures promoted an increase in HUVEC proliferation $(\mathrm{p}<0.001)$ and $1 \mathrm{mM}$ melatonin counteracted this stimulatory effect (Fig. 3A). To study whether the increase in HUVEC proliferation could be due to the release of VEGF by SH-SY5Y cells, the co-culture media were subjected to ELISA to determine VEGF protein levels. The presence of SH-SY5Y cells significantly increased the protein levels of VEGF in the cell co-culture media $(p<0.001)$ whereas the addition of $1 \mathrm{mM}$ melatonin decreased the concentration levels of VEGF and counteracted the stimulatory effect induced by the presence of tumoral cells $(\mathrm{p}<0.05)$ (Fig. 3B). 

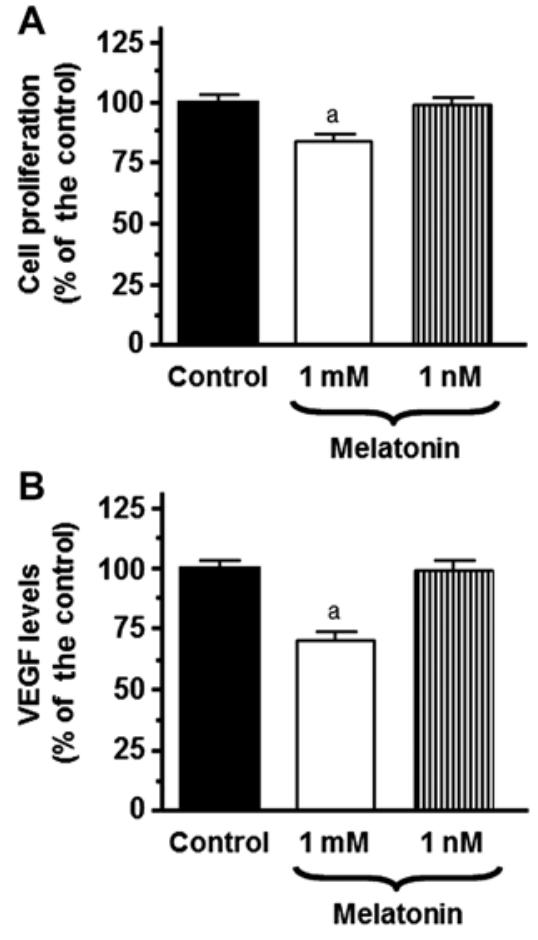

Figure 1. (A) Effects of melatonin $(1 \mathrm{mM}$ and $1 \mathrm{nM})$ or its diluent (ethanol at a $0.001 \%$ final concentration) on SH-SY5Y neuroblastoma cell proliferation assessed using the MTT method. (B) Effects of melatonin $(1 \mathrm{mM}$ and $1 \mathrm{nM}$ ), or its diluent on the protein levels of VEGF in the cell culture media of SH-SY5Y cells as determined by ELISA. Data are expressed as the percentage of the control group (mean $\pm \mathrm{SEM}$ ); ${ }^{\mathrm{a}} \mathrm{p}<0.001$ vs. the other groups. VEGF, vascular endothelial growth factor.
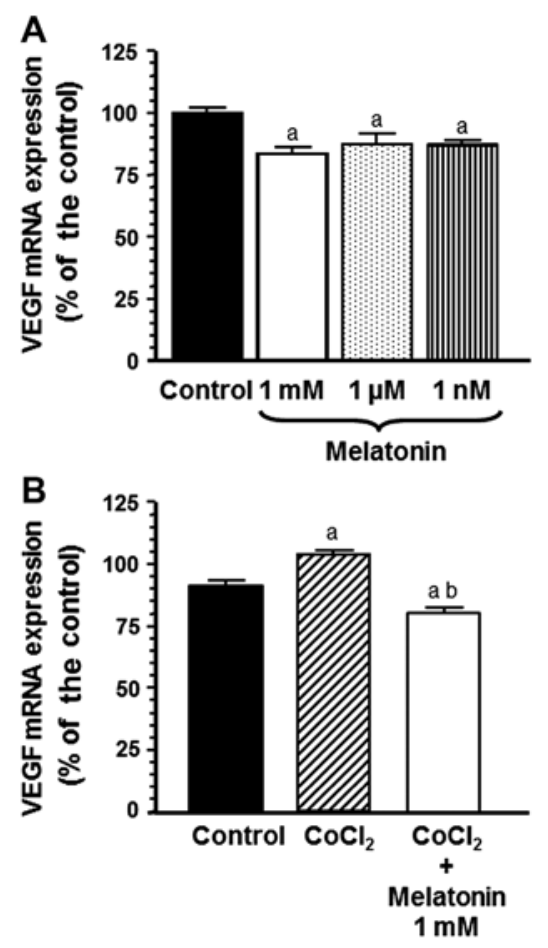

Figure 2. Effects of melatonin on VEGF mRNA expression in SH-SY5Y cells. (A) Cells were incubated with melatonin $(1 \mathrm{mM}, 1 \mu \mathrm{M}$ and $1 \mathrm{nM})$ or ethanol (control) for $4 \mathrm{~h}$. Total mRNA was isolated from cells and reverse-transcribed. The cDNA was subjected to PCR using specific primers for VEGF or s14. (B) Cells were incubated with melatonin $(1 \mathrm{mM})$ and/or $100 \mu \mathrm{M}$ of $\mathrm{CoCl}_{2}$ or ethanol (control) for $4 \mathrm{~h}$. Data are expressed as the percentage of the control group (mean \pm SEM); ${ }^{a} p<0.001$ vs. the control; ${ }^{\mathrm{b}} \mathrm{p}<0.001 \mathrm{vs}$. the $\mathrm{CoCl}_{2}$. VEGF, vascular endothelial growth factor.
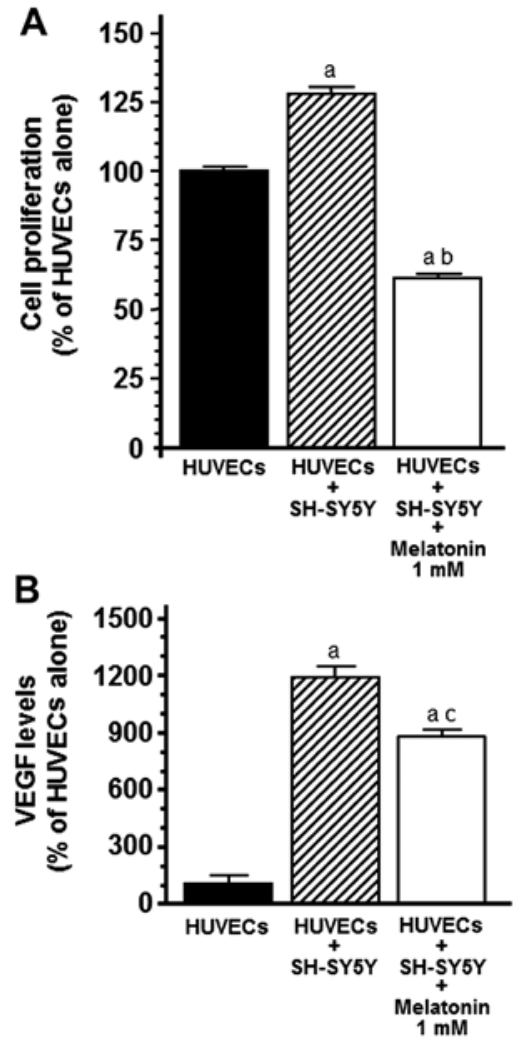

Figure 3. HUVECs were plated $\left(24 \times 10^{3}\right.$ cells/well) on the bottom wells containing VCBM supplemented with $2 \%$ FBS and incubated overnight. Then, SH-SY5Y cells were seeded $\left(35 \times 10^{3}\right.$ cells $)$ on the permeable membrane $(0.45 \mu \mathrm{m})$ of tissue-culture inserts containing VCBM supplemented with $2 \%$ FBS for $24 \mathrm{~h}$. The media were then changed to serum-free VCBM for $72 \mathrm{~h}$ containing melatonin $(1 \mathrm{mM})$ or vehicle (ethanol). At the end of the experiment, cells in the bottom plate were evaluated for proliferative indices using the MTT method and the media were collected, centrifuged and subjected to assessment of the protein levels of VEGF. (A) The effects of melatonin $(1 \mathrm{mM})$ on HUVEC proliferation, in the presence of SH-SY5Y cells in the culture. Data are expressed as the percentage of the control group, cultures containing only HUVECs (mean \pm SEM). (B) The effects of melatonin $(1 \mathrm{mM})$ on the protein levels of VEGF in the co-culture of HUVECs and SH-SY5Y cells. Data are expressed as the percentage of the control group, cultures containing only HUVECs (mean \pm SEM); ${ }^{a} p<0.001$ vs. the HUVECs; ${ }^{b} p<0.001$ vs. the HUVECs + SH-SY5Y; ${ }^{c} p<0.05$ vs. the HUVECs + SH-SY5Y. HUVECs, human umbilical vein endothelial cells; VEGF, vascular endothelial growth factor.

Effects of melatonin on the paracrine activity of VEGF produced by SH-SY5Y cells on HUVEC proliferation. To demonstrate that VEGF produced by tumor cells is angiogenically active and stimulates endothelial cell proliferation, serum-free conditioned media from SH-SY5Y cells were collected and subsequently added to HUVECs to assess their proliferative response. When the conditioned media from the SH-SY5Y cells were added to the cultures, HUVEC growth was markedly stimulated $(\mathrm{p}<0.001)$ and this effect was significantly $(\mathrm{p}<0.001)$ counteracted by the addition of either the anti-VEGF antibody (150 ng/ml) or $1 \mathrm{mM}$ melatonin (Fig. 4).

Effects of melatonin on endothelial cell migration. Since endothelial cell migration is essential for the formation of new blood vessels during neo-angiogenesis, we next studied the effects of melatonin treatment on the migratory properties of endothelial cells using wound-healing assays. We studied whether VEGF contained in the conditioned media collected 


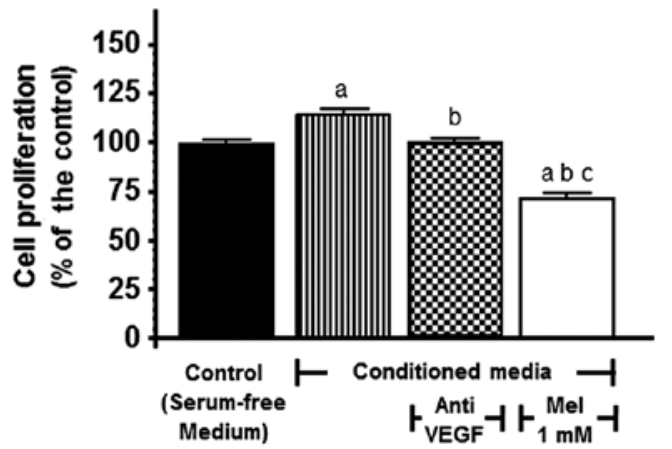

Figure 4. Effects of melatonin on the paracrine activity of VEGF produced by SH-SY5Y cells on HUVEC proliferation. HUVECs were cultured in 96-well multiplates containing VCBM supplemented with $2 \%$ FBS overnight. Cells were then washed twice with PBS and the media were changed to serum-free VCBM containing the conditioned media from the SH-SY5Y cells supplemented with $150 \mathrm{ng} / \mathrm{ml}$ of the anti-human VEGF antibody or $1 \mathrm{mM}$ of melatonin. The cells were cultured for $24 \mathrm{~h}$ and cellular proliferation was assessed using the MTT method. Data are expressed as the percentage of the control group, cultures containing only HUVECs (mean \pm SEM); ${ }^{a} \mathrm{p}<0.001$ vs. the control; ${ }^{b} \mathrm{p}<0.001$ vs. the conditioned media; ${ }^{c} \mathrm{p}<0.001$ vs. the conditioned media + anti-VEGF. HUVECs, human umbilical vein endothelial cells; VEGF, vascular endothelial growth factor.

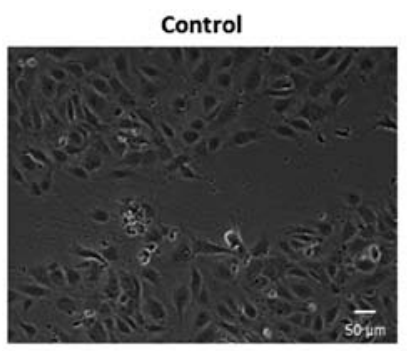

$\mathrm{CM}+$ Melatonin $1 \mathrm{mM}$
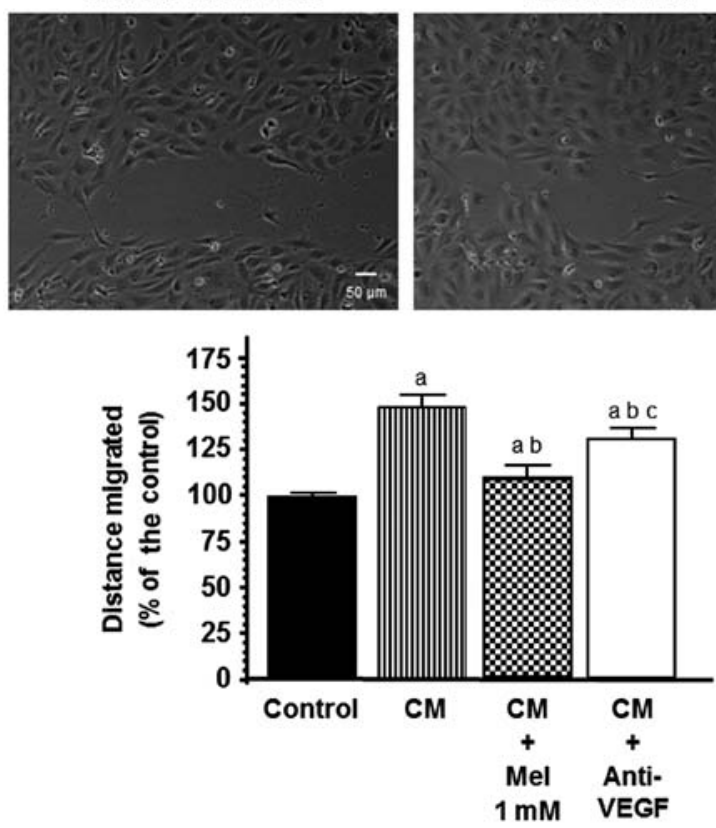

Figure 5. Effects of melatonin $(1 \mathrm{mM})$ and anti-VEGF $(150 \mathrm{ng} / \mathrm{ml})$ on endothelial cell migration induced by the CM collected from the SH-SY5Y cells and analyzed with the wound healing assay. Representative photomicrographs after $6.5 \mathrm{~h}$ of experimentation are shown at a magnification of x10 and the migratory-distance was measured as detailed in the Materials and methods. The migratory distance data shown are expressed as the mean \pm SEM of three experiments; ${ }^{\mathrm{a}} \mathrm{p}<0.001$ vs. the control; ${ }^{\mathrm{b}} \mathrm{p}<0.001$ vs. the $\mathrm{CM} ;{ }^{\mathrm{c}} \mathrm{p}<0.001$ vs. the $\mathrm{CM}+$ melatonin $1 \mathrm{mM}$. HUVECs, human umbilical vein endothelial cells; CM, conditioned media.

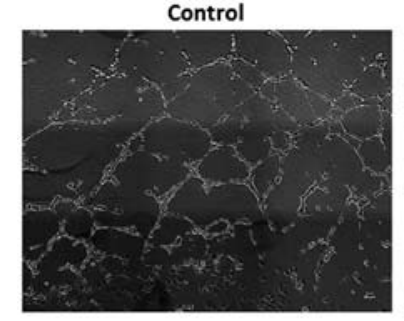

$\mathrm{CM}+$ Melatonin $1 \mathrm{mM}$
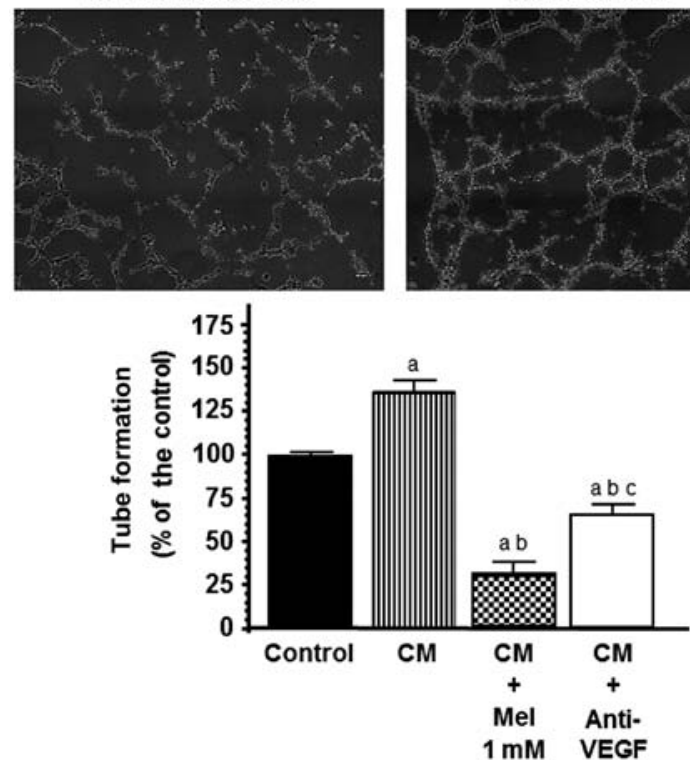

$\mathrm{CM}$

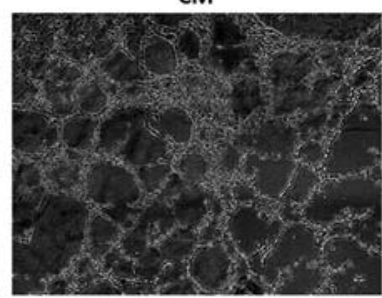

CM+ Anti-VEGF

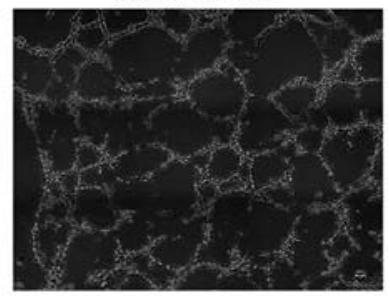

$1 \mathrm{mM}$ VEGF
Figure 6. Effects of melatonin on the tube formation of HUVECs induced by the CM collected from the SH-SY5Y cells. HUVECs were seeded in medium with $2 \%$ FBS containing either SH-SY5Y conditioned media alone or supplemented with $150 \mathrm{ng} / \mathrm{ml}$ anti-VEGF or $1 \mathrm{mM}$ melatonin. After $5 \mathrm{~h}$, the tubular structures were photographed and tubule formation was asessed as described in the Materials and methods. Tubular-formation data are expressed as the percentage of the control group (mean \pm SEM); ${ }^{a} \mathrm{p}<0.01$ vs. the control; ${ }^{\mathrm{b}} \mathrm{p}<0.001$ vs. the $\mathrm{CM} ;{ }^{\mathrm{c}} \mathrm{p}<0.001$ vs. the $\mathrm{CM}+$ melatonin $1 \mathrm{mM}$. HUVECs, human umbilical vein endothelial cells; $\mathrm{CM}$, conditioned media.

from the SH-SY5Y cells was angiogenically active and able to increase the migration of HUVECs and whether melatonin was able to counteract this effect. Clearly, when conditioned media from the SH-SY5Y cells were added to the cultures the migration of HUVECs was markedly stimulated $(p<0.001)$ and this effect was significantly counteracted by the addition of either anti-VEGF (150 ng/ml) or melatonin $1 \mathrm{mM}$ (Fig. 5).

Effects of melatonin on HUVEC capillary structure formation. To study the effects of melatonin on the formation of tubular structures by endothelial cells, forming a complex network of vessels and capillaries, we used an in vitro angiogenesis tube formation assay where we assessed: i) whether VEGF contained in the conditioned media collected from the SH-SY5Y cells was angiogenically active and able to increase the tube formation of HUVECs; and ii) whether melatonin was able to counteract this effect. As shown in Fig. 6, HUVECs plated on reduced growth factor-BME, formed a massive network of tubes after $5 \mathrm{~h}$. When the conditioned media from the SH-SY5Y cells were added to the cultures, tube formation was markedly stimulated and this effect was significantly $(\mathrm{p}<0.001)$ counteracted by the addition of either the anti-VEGF antibody $(150 \mathrm{ng} / \mathrm{ml})$ or melatonin $1 \mathrm{mM}$ (Fig. 6). 


\section{Discussion}

Neuroblastoma is the most common pediatric extracranial and heterogeneous solid tumor in children. It is a highly vascular and aggressive tumor that originates from the neural crest. Angiogenesis, genetic abnormalities and oncogene amplification are mainly responsible for the malignant phenotype of this tumor (1). New blood vessel formation, which supplies vital nutrients to the growing tumor, is regulated by different growth factors, and VEGF is one of the most effective. VEGF is a critical mitogen that regulates growth, neovascularization and migration of endothelial cells in addition, it is expressed and secreted by many neuroblastoma cell lines and primary tumors that contribute to the growth of endothelium in vitro and angiogenesis in vivo leading to poor prognosis in high-risk neuroblastoma $(1,25)$. SH-SY5Y is a human cell line, cloned from a bone marrow biopsy-derived line obtained from a four-year old female with neuroblastoma, called SK-N-SH and first reported in 1973 (26). It has been demonstrated that VEGF produced from tumor cells is essential for the progression of experimental neuroblastoma and may function in both paracrine and autocrine manners to allow tumor expansion and growth in the SH-SY5Y neuroblastoma cell line (25). At present, the role of melatonin in the regulation of cancer cell growth, more particularly in estrogen-dependent tumors, is well established $(9-11,14,16,17,27)$. Lissoni et al (28) were the first to suggest that melatonin could exert anti-angiogenic effects in cancer and they also demonstrated a decrease in the serum levels of VEGF in cancer patients treated with melatonin. The effects of melatonin on angiogenesis have been studied in pancreatic, colon, breast, lung, hepatocellular and cervical cancer $(23,29-32)$. In the present study, our objective was to demonstrate the usefulness of melatonin against the pro-angiogenic effects of either VEGF or the conditioned media from human neuroblastoma cells (SH-SY5Y).

The present study demonstrated that melatonin treatment has anti-angiogenic activity at different steps of the angiogenic process in SH-SY5Y cells. Melatonin inhibited the pro-angiogenic effects of SH-SY5Y cells and decreased the pro-angiogenic effects of the conditioned media from the SH-SY5Y cells stimulating proliferation, migration and tubular network formation by endothelial cells. After disruption of the vessel wall, endothelial cells must adhere to the extracellular matrix in the stromal space, migrate out from the original vascular structure, proliferate and organize into new vascular structures. Each of these processes was simulated in part by one of the in vitro assays used. Melatonin at a concentration of $1 \mathrm{mM}$ had an inhibitory effect on cell proliferation of the neuroblastoma cell line. SH-SY5Y cells express melatonin MT1 receptor and different aspects related with their growth are modulated by this indolamine; thus, melatonin induces apoptosis (33), histone hyperacetylation (34) or anti-neuroinflammatory effects (35) in this neuroblastoma cell line. The effects of melatonin on cancer cells depend on different factors, such as its concentration, duration of exposure and the characteristics of the cells assessed. In the present study, only pharmacological concentrations of melatonin were effective. In previous studies $(10-12,17,36)$, a strong inhibitory effect induced by nanomolar concentrations of melatonin on human breast cancer cells has been described.
However, high concentrations of melatonin are necessary to obtain oncostatic effects in many other types of normal and cancer cells $(31,33,37-39)$. Melatonin is a highly lipid-soluble indolamine which may easily cross the blood-brain barrier, and there is evidence that melatonin concentration in the cerebrospinal fluid is higher than in blood (40). Melatonin is also three orders of magnitude more concentrated in neoplastic and adipose tissue of the breast (41). These high concentrations of melatonin in some tissues may explain why high levels of melatonin are necessary to achieve oncostatic effects.

Since endothelial cell migration/invasion is essential for the formation of new blood vessels during neo-angiogenesis, we next studied the regulatory effects of melatonin on the conditioned media collected from the SH-SY5Y cells, which are angiogenically active and able to increase the migration of endothelial cells. Thus, the conditioned media from SH-SY5Y cells stimulated the migration of endothelial cells and this effect was significantly counteracted by the addition of either anti-VEGF or $1 \mathrm{mM}$ of melatonin. These results suggest that VEGF produced by tumor cells could be responsible for inducing a proliferative response of endothelial cells.

Another important step during neo-angiogenesis is the formation of tubes by endothelial cells forming a complex network of vessels and capillaries $(2,42,43)$. To study the effects of melatonin on this biological event we used an endothelial cell capillary-like tube formation assay. Endothelial cells plated on a basement membrane extract formed a massive network of tubes. Conditioned medium collected from the neuroblastoma cells was angiogenically active and stimulated tube formation. This effect was significantly counteracted by the addition of either the anti-VEGF antibody or melatonin, which suggests that melatonin-decreased capillary structure formation stimulated by the conditioned media from the SH-SY5Y cells, may occur through the inhibition of VEGF. Our results support the hypothesis that melatonin decreases the proliferation, migration and tube formation of endothelial cells by regulating the production of VEGF by neuroblastoma cells. We found that the same concentration of melatonin that inhibits the proliferation of SH-SY5Y cells also decreases the levels of VEGF in neuroblastoma cell-culture media. Previous studies have described a decrease in the production of VEGF protein induced by melatonin in human pancreatic carcinoma cells (PANC-1), human alveolar adenocarcinoma cells (A549) and human breast cancer cells (MCF-7) $(29,31,32)$. With the aim of determining whether this inhibitory effect of melatonin on VEGF production was due to a downregulation of the VEGF mRNA expression in SH-SY5Y cells, we then incubated SH-SY5Y cells with different melatonin concentrations. The results obtained demonstrate that melatonin did in fact downregulate VEGF mRNA expression. SH-SY5Y cells were also exposed to cobalt chloride $\left(\mathrm{CoCl}_{2}\right)$ to mimick hypoxia, a well-known inducer of VEGF mRNA expression, and the addition of $1 \mathrm{mM}$ of melatonin significantly counteracted the stimulatory effect of $\mathrm{CoCl}_{2}$ on VEGF mRNA gene expression. It has been shown that the pharmacological concentrations of melatonin also suppress the VEGF mRNA expression induced by cobalt chloride in other tumor cells, such as human pancreatic carcinoma (PANC-1), human breast cancer (MCF-7) and human alveolar adenocarcinoma cells (A549) $(29,31,32)$. In these tumor cells, melatonin also decreases hypoxia-inducible 
factor (HIF)-1 $\alpha$ protein levels, suggesting a role for transcription factor HIF-1 $\alpha$ in the suppression of VEGF expression $(29,30)$. In the present study, we developed a co-culture system of HUVECs and SH-SY5Y cells to investigate whether neuroblastoma cells affect the growth of the endothelial cells. We found that HUVECs exhibited increased cell proliferation when they were co-cultured with neuroblastoma cells and this process was prevented when melatonin was added to the culture medium. These results suggested that the local VEGF produced by tumor cells could be responsible for the proliferative response of endothelial cells. Undoubtedly, the presence of neuroblastoma cells increased the protein levels of VEGF in the cell co-culture media and the addition of melatonin decreased the concentration levels of VEGF and counteracted the stimulatory effect induced by the presence of neuroblastoma cells. In addition, to demonstrate the paracrine effects of neuroblastoma-secreted VEGF on HUVEC growth, we studied whether VEGF within the conditioned medium collected from the neuroblastoma cell cultures was able to increase the proliferation of HUVECs. When the conditioned medium was added to the HUVECs in the culture, their growth was stimulated. The proliferative response of the HUVECs was abolished in the presence of an anti-VEGF antibody, indicating that VEGF produced by the neuroblastoma cells was the major factor that induces cellular proliferation in endothelial cells. When melatonin was added to the HUVEC cultures in the presence of the conditioned medium collected from the neuroblastoma cells, the stimulatory effect of the conditioned medium was also counteracted by this indolamine.

The present study, describes the anti-angiogenic effects of melatonin in relation to neuroblastoma. The anti-angiogenic effects of melatonin through its inhibitory effects on VEGF have also been described in human pancreatic carcinoma cells (PANC-1), human alveolar adenocarcinoma (A549), human colon cancer (HCT116), human breast cancer (MCF-7), cervical cancer cells (HeLa) and adenocarcinomic human alveolar basal epithelial cells (29-32). All these findings support a model in which neuroblastoma cells produce VEGF that functions in a paracrine manner to increase proliferation, invasion, migration and tube formation of endothelial cells. Melatonin may play a role in the paracrine interactions between neuroblastoma cells and proximal endothelial cells through the downregulation of the expression of VEGF in SH-SY5Y cells, thus decreasing the levels of VEGF around endothelial cells. Lower levels of VEGF could be important in decreasing endothelial cell proliferation, invasion, migration and tube formation. Collectively, our findings suggest that the anti-angiogenic effects of melatonin provide a promising therapeutic approach for the treatment of neuroblastoma.

\section{Acknowledgements}

We are grateful to Dr María Elsa Valdizán from the Pharmacology Division of the Department of Physiology and Pharmacology of the University of Cantabria (Spain), for the neuroblastoma cells (SH-SY5Y) which were a kind gift. The present study was supported by grants from the Spanish Ministry of Science, Technology and Innovation (SAF2013-42012-P) and from the Instituto de Investigación Sanitaria Valdecilla (IDIVAL) (APG/12).

\section{References}

1. Roy Choudhury S, Karmakar S, Banik NL and Ray SK: Targeting angiogenesis for controlling neuroblastoma. J Oncol 2012: $782020,2012$.

2. Bareschino MA, Schettino C, Colantuoni G, Rossi E, Rossi A, Maione P, Ciardiello F and Gridelli C: The role of antiangiogenetic agents in the treatment of breast cancer. Curr Med Chem 18: 5022-5032, 2011.

3. Lyons J III, Anthony CT and Woltering EA: The role of angiogenesis in neuroendocrine tumors. Endocrinol Metab Clin North Am 39: 839-852, 2010.

4. Rössler J, Monnet Y, Farace F, Opolon P, Daudigeos-Dubus E, Bourredjem A, Vassal G and Geoerger B: The selective VEGFR1-3 inhibitor axitinib (AG-013736) shows antitumor activity in human neuroblastoma xenografts. Int J Cancer 128: 2748-2758, 2011

5. Cook KM and Figg WD: Angiogenesis inhibitors: Current strategies and future prospects. CA Cancer J Clin 60: 222-243, 2010.

6. Liang Y and Hyder SM: Proliferation of endothelial and tumor epithelial cells by progestin-induced vascular endothelial growth factor from human breast cancer cells: Paracrine and autocrine effects. Endocrinology 146: 3632-3641, 2005.

7. Brignole C, Marimpietri D, Pastorino F, Nico B, Di Paolo D, Cioni M, Piccardi F, Cilli M, Pezzolo A, Corrias MV, et al: Effect of bortezomib on human neuroblastoma cell growth, apoptosis, and angiogenesis. J Natl Cancer Inst 98: 1142-1157, 2006.

8. Segerström L, Fuchs D, Bäckman U, Holmquist K, Christofferson R and Azarbayjani F: The anti-VEGF antibody bevacizumab potently reduces the growth rate of high-risk neuroblastoma xenografts. Pediatr Res 60: 576-581, 2006.

9. Blask DE, Sauer LA and Dauchy RT: Melatonin as a chronobiotic/anticancer agent: Cellular, biochemical, and molecular mechanisms of action and their implications for circadian-based cancer therapy. Curr Top Med Chem 2: 113-132, 2002.

10. Cos S and Sánchez-Barceló EJ: Melatonin and mammary pathological growth. Front Neuroendocrinol 21: 133-170, 2000.

11. Cos S and Sánchez-Barceló EJ: Melatonin, experimental basis for a possible application in breast cancer prevention and treatment. Histol Histopathol 15: 637-647, 2000.

12. Hill SM and Blask DE: Effects of the pineal hormone melatonin on the proliferation and morphological characteristics of human breast cancer cells (MCF-7) in culture. Cancer Res 48: 6121-6126, 1988.

13. Cos S, Fernández R, Güiézmes A and Sánchez-Barceló EJ: Influence of melatonin on invasive and metastatic properties of MCF-7 human breast cancer cells. Cancer Res 58: 4383-4390, 1998.

14. Cos S, González A, Martínez-Campa C, Mediavilla MD, Alonso-González C and Sánchez-Barceló EJ: Estrogen-signaling pathway: A link between breast cancer and melatonin oncostatic actions. Cancer Detect Prev 30: 118-128, 2006.

15. Alonso-González C, González A, Mazarrasa O, Güezmes A, Sánchez-Mateos S, Martínez-Campa C, Cos S, Sánchez-Barceló EJ and Mediavilla MD: Melatonin prevents the estrogenic effects of sub-chronic administration of cadmium on mice mammary glands and uterus. J Pineal Res 42: 403-410, 2007.

16. Cos S, González A, Güezmes A, Mediavilla MD, Martínez-Campa C, Alonso-González C and Sánchez-Barceló EJ: Melatonin inhibits the growth of DMBA-induced mammary tumors by decreasing the local biosynthesis of estrogens through the modulation of aromatase activity. Int J Cancer 118: 274-278, 2006.

17. Cos S, González A, Martínez-Campa C, Mediavilla MD, AlonsoGonzález C and Sánchez-Barceló EJ: Melatonin as a selective estrogen enzyme modulator. Curr Cancer Drug Targets 8: 691-702, 2008.

18. González A, Cos S, Martínez-Campa C, Alonso-González C, Sánchez-Mateos S, Mediavilla MD and Sánchez-Barceló EJ: Selective estrogen enzyme modulator actions of melatonin in human breast cancer cells. J Pineal Res 45: 86-92, 2008.

19. Martínez-Campa C, González A, Mediavilla MD, AlonsoGonzález C, Alvarez-García V, Sánchez-Barceló EJ and Cos S: Melatonin inhibits aromatase promoter expression by regulating cyclooxygenases expression and activity in breast cancer cells. Br J Cancer 101: 1613-1619, 2009. 
20. Gerber HP, McMurtrey A, Kowalski J, Yan M, Keyt BA, Dixit V and Ferrara N: Vascular endothelial growth factor regulates endothelial cell survival through the phosphatidylinositol3'-kinase/Akt signal transduction pathway. Requirement for Flk-1/KDR activation. J Biol Chem 273: 30336-30343, 1998.

21. Gingis-Velitski S, Zetser A, Flugelman MY, Vlodavsky I and Ilan N: Heparanase induces endothelial cell migration via protein kinase B/Akt activation. J Biol Chem 279: 23536-23541, 2004

22. Gu Q, Wang D, Wang X, Peng R, Liu J, Jiang T, Wang Z, Wang S and Deng $\mathrm{H}$ : Basic fibroblast growth factor inhibits radiationinduced apoptosis of HUVECs. I. The PI3K/AKT pathway and induction of phosphorylation of BAD. Radiat Res 161: 692-702, 2004.

23. Alvarez-García V, González A, Alonso-González C, Martínez-Campa C and Cos S: Antiangiogenic effects of melatonin in endothelial cell cultures. Microvasc Res 87: 25-33, 2013.

24. Mosmann T: Rapid colorimetric assay for cellular growth and survival: Application to proliferation and cytotoxicity assays. J Immunol Methods 65: 55-63, 1983.

25. Bäckman U, Svensson A and Christofferson R: Importance of vascular endothelial growth factor $A$ in the progression of experimental neuroblastoma. Angiogenesis 5: 267-274, 2002.

26. Biedler JL, Helson L and Spengler BA: Morphology and growth, tumorigenicity, and cytogenetics of human neuroblastoma cells in continuous culture. Cancer Res 33: 2643-2652, 1973.

27. Sánchez-Barceló EJ, Cos S, Mediavilla D, Martínez-Campa C, González A and Alonso-González C: Melatonin-estrogen interactions in breast cancer. J Pineal Res 38: 217-222, 2005.

28. Lissoni P, Rovelli F, Malugani F, Bucovec R, Conti A and Maestroni GJ: Anti-angiogenic activity of melatonin in advanced cancer patients. Neuro Endocrinol Lett 22: 45-47, 2001

29. Dai M, Cui P, Yu M, Han J, Li H and Xiu R: Melatonin modulates the expression of VEGF and HIF-1 $\alpha$ induced by $\mathrm{CoCl}_{2}$ in cultured cancer cells. J Pineal Res 44: 121-126, 2008.

30. Park SY, Jang WJ, Yi EY, Jang JY, Jung Y, Jeong JW and Kim YJ: Melatonin suppresses tumor angiogenesis by inhibiting HIF-1 $\alpha$ stabilization under hypoxia. J Pineal Res 48: 178-184, 2010.

31. Cui P, Yu M, Peng X, Dong L and Yang Z: Melatonin prevents human pancreatic carcinoma cell PANC-1-induced human umbilical vein endothelial cell proliferation and migration by inhibiting vascular endothelial growth factor expression. J Pineal Res 52: 236-243, 2012.
32. Alvarez-García V, González A, Alonso-González C, Martínez-Campa C and Cos S: Regulation of vascular endothelial growth factor by melatonin in human breast cancer cells. J Pineal Res 54: 373-380, 2013.

33. García-Santos G, Antolín I, Herrera F, Martín V, RodríguezBlanco J, del Pilar Carrera M and Rodríguez C: Melatonin induces apoptosis in human neuroblastoma cancer cells. J Pineal Res 41: 130-135, 2006.

34. Pan Y and Niles LP: Epigenetic mechanisms of melatonin action in human SH-SY5Y neuroblastoma cells. Mol Cell Endocrinol 402: 57-63, 2015.

35. Wongprayoon P and Govitrapong P: Melatonin attenuates methamphetamine-induced neuroinflammation through the melatonin receptor in the SH-SY5Y cell line. Neurotoxicology 50: 122-130, 2015.

36. Cos S, Martínez-Campa C, Mediavilla MD and SánchezBarceló EJ: Melatonin modulates aromatase activity in MCF-7 human breast cancer cells. J Pineal Res 38: 136-142, 2005.

37. Sainz RM, Mayo JC, Tan DX, León J, Manchester L and Reiter RJ: Melatonin reduces prostate cancer cell growth leading to neuroendocrine differentiation via a receptor and PKA independent mechanism. Prostate 63: 29-43, 2005.

38. Alvarez-García V, González A, Alonso-González C, Martínez-Campa C and Cos S: Melatonin interferes in the desmoplastic reaction in breast cancer by regulating cytokine production. J Pineal Res 52: 282-290, 2012.

39. González A, Alvarez-García V, Martínez-Campa C, AlonsoGonzález $\mathrm{C}$ and Cos S: Melatonin promotes differentiation of 3T3-L1 fibroblasts. J Pineal Res 52: 12-20, 2012.

40. Longatti P, Perin A, Rizzo V, Comai S, Giusti P and Costa CV: Ventricular cerebrospinal fluid melatonin concentrations investigated with an endoscopic technique. J Pineal Res 42: 113-118, 2007.

41. Maestroni GJ and Conti A: Melatonin in human breast cancer tissue: Association with nuclear grade and estrogen receptor status. Lab Invest 75: 557-561, 1996.

42. Dvorak HF, Weaver VM, Tlsty TD and Bergers G: Tumor microenvironment and progression. J Surg Oncol 103: 468-474, 2011.

43. Staton CA, Stribbling SM, Tazzyman S, Hughes R, Brown NJ and Lewis CE: Current methods for assaying angiogenesis in vitro and in vivo. Int J Exp Pathol 85: 233-248, 2004. 